\title{
Uso dos MCE, MMEEBB e tecnologia computacional no processo de resgate e perenização do conhecimento a idosos e pessoas com atrasos de aprendizado
}

\author{
Mirian C. Silva ${ }^{1}$, Mislene D. Silva ${ }^{1}$, Sara L. de Melo ${ }^{1}$, Thales O. Lima ${ }^{1}$, Stéphane \\ C. Souza ${ }^{2}$, Luciano V. Lima ${ }^{1}$ \\ ${ }^{1}$ Departamento de Engenharia Elétrica \\ Universidade Federal de Uberlândia - UFU \\ Uberlândia - MG - Brasil \\ ${ }^{2}$ Centro Universitário de Patos de Minas - UNIPAM \\ Patos de Minas - MG - Brasil \\ \{ miriancamila0, mislenedalila, saraluziamelo, spsouza72, lucianovieiralimaster\}@ gmail.com
}

\begin{abstract}
This is paper consists in presenting the structuring of a multiplatform application with tools that helps both people with attention deficit hyperactivity disorder motivated to learn, and elderly people with Alzheimer's disease to train their brains allowing the effective memory of knowledge and thus softening the effects of the disease. In this way, it aims to ensure that the individual, in abstracting a new knowledge, will not forget the previous one. The results obtained in this study indicate that the developed application confirms the positive effect of the use of computational technologies to aid the learning of individuals, as well as the use of MCE and $M M E E B B$ in these technologies.
\end{abstract}

Resumo. Este trabalho consiste em apresentar a estruturação de um aplicativo multiplataforma com ferramentas que auxilie tanto pessoas com transtorno de déficit de atenção e hiperatividade motivadas a aprender, quanto idosos com doença de Alzheimer a treinarem seu cérebro permitindo a memorização efetiva do conhecimento e com isso amenizando os efeitos da doença. Dessa forma, visa-se garantir que o indivíduo, ao abstrair um novo conhecimento, não irá esquecer o anterior. Os resultados alcançados neste estudo indicam que, o aplicativo desenvolvido vem confirmando o efeito positivo do uso das tecnologias computacionais para auxiliar ao aprendizado dos indivíduos, e também do uso dos MCE e do MMEEBB nestas tecnologias.

\section{Introdução}

Atualmente, com os avanços tecnológicos, alguns indivíudos não utilizam sua capacidade cognitiva de maneira eficaz ou não são estimulados da maneira correta para tal. A evolução dessas tecnologias afetam a maneira de focar a atenção de alunos em sala de aula. Por exemplo, deixa de ser atrativo em relação aos bombardeios de informações que recebem de celulares cada vez mais modernos, deixando ultrapassada a tradicional maneira de lecionar e colocando a necessidade de recorrer a mudanças que proporcionam métodos para melhor aproveitamento dos alunos. 
VII Congresso Brasileiro de Informática na Educação (CBIE 2018)

Anais do XXIX Simpósio Brasileiro de Informática na Educação (SBIE 2018)

Para a maioria dos estudantes que recebem testes de avaliação de ensino no fim de algum período, relembrar informações que já foram vistas há certo tempo é muito difícil. Isso acontece devido ao cérebro humano armazenar poucas informações em curto prazo e perder informações que não são repetidas em longo prazo. [Ferreira, 2014].

Se para a maioria dos alunos, que estão nos $90 \%$ da Curva de Gauss, existe uma dificuldade para absorver com qualidade o conteúdo ministrado. Para aqueles com Transtorno de Déficit de Atenção e Hiperatividade - TDAH é quase impossível. Diante desse sistema escolar, o aluno não consegue se concentrar e é deixado "para trás" com assuntos que não são aprendidos e que ficam acumulados, sem estímulo do seu raciocínio.

Normalmente, pessoas com TDAH são tratadas com preconceito, pois possuem uma deficiência "invisível" aos demais indivíduos, sendo vistas como mal-educadas ou até mesmo preguiçosas ou sem interesse em aprender.

Outras vertentes preocupantes são os idosos e principalmente aqueles diagnosticados com doença de Alzheimer, que apresentam grande dificuldade de memorização. Conforme [Nitrini, 1993] após os 85 anos há uma maior chance de desenvolver a doença. Já para o [Instituto Alzheimer Brasil, 2018] a prevalência é de $70 \%$ do total de casos.

Segundo [Silva, 2017] uma forma de assegurar a recordação do aprendizado de forma eficaz e eficiente durante semanas ou anos é utilizar o Método de Memorização Exponencial Efetivo na Base Binária - MMEEBB que contém a Curva de Memorização Efetiva - CME originada pelo Intervalo de Recordação do Aprendizado - IRA.

Portanto o objetivo deste trabalho consiste em apresentar o desenvolvimento de um aplicativo mobile com ferramentas que auxilie, tanto pessoas com TDAH motivadas a aprender, quanto idosos com doença de Alzheimer a treinarem seu cérebro permitindo a memorização efetiva do conhecimento através do MMEEBB e com isso amenizando os efeitos da doença. Dessa forma, objetiva-se garantir que o indivíduo, ao abstrair um novo conhecimento, não irá esquecer o anterior.

\section{Trabalhos Correlatos}

Esta seção contém informações norteadoras do trabalho, através de fundamentos da bibliografia publicada sobre o assunto.

\subsection{Transtorno de déficit de atenção e hiperatividade - TDAH}

Transtorno de déficit de atenção e hiperatividade é uma doença neuropsiquiátrica crônica caracterizada por desatenção excessiva, inquietação, impulsividade, desorganização entre outras que se inicia na infância, mas pode acompanhar o indivíduo ao longo do seu desenvolvimento [Barkley, 2008].

Algumas pessoas, tanto adulto quanto criança apresentam algum nível dessas características, o que é considerado completamente normal. Porém quando elas passam a causar problemas intensos é preciso uma avaliação médica para diagnosticar TDAH, para iniciar o tratamento que pode prevenir e aliviar o sofrimento [Amorim, 2018]. 
VII Congresso Brasileiro de Informática na Educação (CBIE 2018)

Anais do XXIX Simpósio Brasileiro de Informática na Educação (SBIE 2018)

Frequentemente, em práticas de estudo, de leitura ou outras que requeiram concentração, há uma elevação dos níveis de ativação no cérebro, por causa destas condições. Em ocorrências típicas de TDAH, o que mais acontece a nível psicofisiológico é a hipofunção/hipoativação do córtex pré-frontal, em que uma fração considerável de neurônios pulsam lentamente, principalmente quando a situação requer mais estímulo mental [Amorim, 2018].

Na Figura 1, são apresentadas três imagens sobre o comportamento do cérebro. A Figura 1 (a) exibe um cérebro normal em repouso. Na Figura 1 (b), representa o cérebro de um indivíduo com TDAH em repouso. As regiões de mais baixo estímulo (com forma de buracos) apontam menos gasto de energia (baixo metabolismo de glicose). Nota-se uma semelhança entre o cérebro de uma pessoa com TDAH com a de uma pessoa normal em repouso.

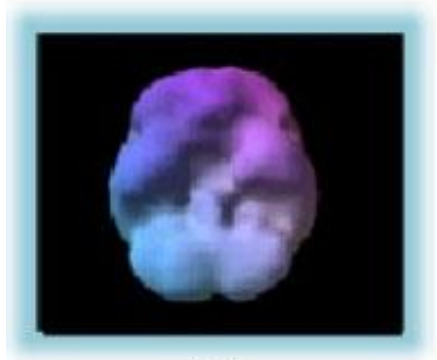

(a)

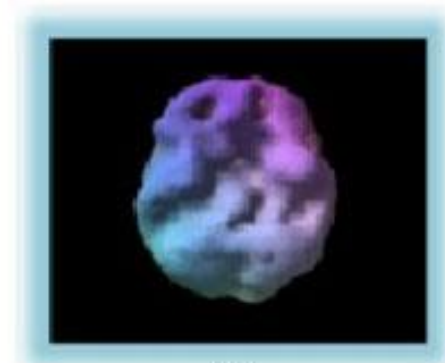

(b)

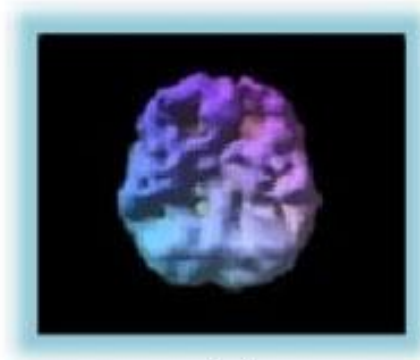

(c)

Figura 1. Comportamento do cérebro

O problema surge mais nitidamente quando o cérebro necessita de concentração, esforço mental e domínio da hiperatividade, pela insuficiência de uma plena ativação cognitiva. Na Figura 1 (c), mostra claramente este acontecimento, apontando que pessoas portadoras de TDAH defrontam os maiores obstáculos precisamente no instante em que necessitam de concentração [Amorim, 2018].

A síndrome é distribuída em três tipos: TDAH com sintomas de desatenção; TDAH com sintomas de hiperatividade/impulsividade; TDAH mesclado, manifestando uma predominância a morbidades, bem como a transtornos disruptivos do comportamento [Amorim, 2018].

O procedimento de avaliação diagnóstica é extenso, incluindo obrigatoriamente a coleta de informações com os pais, com o indivíduo e com a escola. A terapêutica do TDAH abrange uma abordagem complexa, contendo ações psicossociais e psicofarmacológicas. A medicação com maior eficácia comprovada foi o metilfenidato [Amorim, 2018].

Já [Bastos et al.,2012] propôs o desenvolvimento de um jogo de supermercado para avaliar a cognição do Transtorno de Déficit de Atenção/Hiperatividade de crianças e adolescentes. Os resultados da pesquisa se mostraram promissores quanto à utilização do jogo, diagnosticando casos de TDHA na população analisada. 
VII Congresso Brasileiro de Informática na Educação (CBIE 2018)

Anais do XXIX Simpósio Brasileiro de Informática na Educação (SBIE 2018)

\subsection{Alzheimer}

Alzheimer é uma doença neurodegenerativa cerebral que provoca progressiva perda da capacidade cognitiva (memória, linguagem, atenção e orientação), deterioração das funções da memória e perda da habilidade de cuidar de si próprio [Filho, 2017].

Essas deficiências ocorrem devido a mortes neural que acontecem decorrente de uma falha na metabolização de duas proteínas dentro do cérebro: a chamada proteína precursora do amiloide, que está presente fora do neurônio, e a proteína tau, que faz parte do citoesqueleto celular. A formação de emaranhados neurofibrilares (decorrentes da hiperfosforilação da proteína tau) e os depósitos de substância amiloide geram disfunção das células nervosas, efeito tóxico celular e morte neural [Bacelar, 2009].

Conforme informações da Associação Brasileira de Alzheimer, no Brasil possui aproximadamente 15 milhões de indivíduos com mais de 60 anos de idade, e dentre estes 6\% são portadores de Alzheimer. Nos Estados Unidos da América (EUA), esta doença é a quarta que mais mata pessoas idosas entre 75 e 80 anos, perdendo apenas para o infarto, o derrame e o cancer [Abraz, 2018].

Essa enfermidade é prevalente na terceira idade, no entanto, muitos indivíduos com 70 anos ou mais não apresentam sintomas. A inteligência, hábitos de trabalho bem organizados, raciocínio e altos níveis educacionais compensam muitas deficiências progressivas da idade. Quanto maior o número de desafios intelectuais em que o cérebro for submetido, mais numerosas serão as conexões sinápticas (entre neurônios) e melhor serão aproveitados os novos neurônios diariamente produzidos. Essa "reserva cognitiva" desacelera o declínio das funções cerebrais na idade avançada [Bacelar, 2009].

Se a doença de Alzheimer for diagnosticada no começo, é factível retardar seu avanço, bem como um maior monitoramento dos sintomas e consequentemente uma melhor qualidade de vida tanto para o indivíduo com Alzheimer, quanto para a família [Bacelar, 2009].

\subsection{Método de Memorização Exponencial Efetiva na Base Binária - MMEEBB}

O MMEEBB estabelece Intervalos de Reforço do Aprendizado - IRA definido por meio da Curva de Memorização Efetiva - CME. A CME é relevante para informar a pessoa quando ela necessita lembrar do conhecimento [Silva, 2017].

Esta metodologia tem o propósito de utilizar métodos de memorização dos conhecimentos adquiridos, a fim de que eles não sejam esquecidos e abandonados em um extenso período. Sendo repetido não somente através da leitura de sínteses, ou por meio da releitura de obras ou da revisão das atividades, mas também empregando a informação obtida, através de discussões em bate-papos com assuntos afins. A pessoa que escolher esta técnica de aprendizagem necessita seguir precisamente o período temporal total, onde a cada período, o assunto deverá ser revisado [Silva, 2017].

\subsection{Tecnologia na educação e na saúde}

Quando se trata de aulas no modelo "tradicional", é possível ver o descontentamento e o desinteresse nos olhares da maioria. Isso acontece porque o nosso cérebro consegue assimilar melhor o conteúdo diante de imagens, áudios, vídeos e não apenas centenas de palavras lançadas ao quadro-negro. Isso torna as aulas mais 
VII Congresso Brasileiro de Informática na Educação (CBIE 2018)

Anais do XXIX Simpósio Brasileiro de Informática na Educação (SBIE 2018)

interessantes, dinâmicas e interativas. Por esse motivo instituições de ensino têm procurado cada dia mais modernizar as formas de ensino com o auxílio da tecnologia. Existem hoje no mercado softwares, programas, aplicativos e até jogos que auxiliam no aprendizado [Idoeta, 2014].

As mudanças ocorridas pelos avanços tecnológicos e o crescente uso da web na vida cotidiana ocasiona para os professores a necessidade de adquirir novos conhecimentos e novas posturas para ensinar. É preciso entender as novas tecnologias não somente para bem aplicá-las, mas também, para saber orientar os estudantes, explorando ao máximo sua capacidade e as potencialidades das ferramentas digitais [Idoeta, 2014].

Ainda assim, em nenhum outro setor o impacto da tecnologia é mais aparente quanto na saúde, com constantes pesquisas para desenvolvimento de tecnologias cada vez mais avançadas para tratamentos eficazes. Pesquisas avaliam que no mínimo um terço dos gastos na saúde é devido às novas tecnologias existentes em todo o âmbito da medicina, de prevenção, de diagnóstico, de tratamento e de reabilitação [Collucci, 2015].

No mercado existem utilitários que propiciam a prevenção de doenças, a adesão de indivíduos aos tratamentos, a disponibilização de prontuários eletrônicos, a produção de robôs que realizam cirurgias com pouco sangramento e com rápida recuperação, $o$ aperfeiçoamento da tecnologia biomédica, entre outros [Collucci, 2015].

Progressivamente na área da saúde, estão sendo inseridas tecnologias direcionadas para a recuperação e tratamento das pessoas. São exemplos: os aparelhos de reabilitação, o emprego de jogos e a realidade virtual. Desta forma, cada vez mais, acontece uma melhora na qualidade de vida tanto dos pacientes, quanto de seus familiares, diminuindo a espera do tratamento, dos resultados, das internações e da recuperação de várias doenças. Enfim, a evolução tecnológica no âmbito da saúde, está sendo primordial para o seu avanço bem como para o cuidado e bem-estar do paciente [Collucci, 2015].

\subsection{Mapa de Conhecimento Estruturado - MCE}

A organização dos MCE aconselha o indivíduo a não receber um novo conhecimento sem que primeiro tenha compreendido $100 \%$ do conhecimento anterior. O fragmento de cada assunto é separado em etapas com informações antecedentes e sequentes, onde cada novo assunto é interpretado por um raio de conheciemtno no qual a área gerada por este raio retrata a ignorância que terá de ser resolvida. É importante salientar que uma área de ignorância não concluída, atenua o interesse do estudante levando o abandono em relação ao estudo do conteúdo [Ferreira, 2014].

Desta maneira, um dos tópicos importantes dos MCE é que eles estruturam de maneira personalizada o método de aprendizagem do estudante. Certificando que, a cada adição de um raio de conhecimento, há um entendimento pleno sobre um certo assunto, sustentando a categoria aluno envolvida em permanecer estudando e tornando-se capacitado a praticar atividades com o estudo adquirido [Ferreira, 2014]. 
VII Congresso Brasileiro de Informática na Educação (CBIE 2018)

Anais do XXIX Simpósio Brasileiro de Informática na Educação (SBIE 2018)

\section{Metodologia}

Esta pesquisa é de natureza exploratória descritiva, com a finalidade de analisar, detalhar e exibir as proporções de um instrumento de estudo. $\mathrm{O}$ presente trabalho descreve a criação e desenvolvimento de um aplicativo multiplataforma para auxiliar os indivíduos que possuem TDAH a reter o conhecimento em sua memória e consequentemente retardando a doença de Alzheimer em idosos através do MMEEBB que enviará notificações sobre o que necessita recordar, para que a informação fique consolidada em sua memória.

Foi realizado o levantamento e estudo dos requisitos necessários para a construção do aplicativo através de artigos científicos, livros e sites relacionados ao tema.

Posteriormente efetuou-se a seleção de todas as informações que estarão contidas no aplicativo para o idoso com Alzheimer e também para o estudante com TDAH, bem como a escolha das disciplinas de um curso para a realização deste estudo. O próximo passo foi a criação dos protótipos do utilitário. Posteriormente, ocorreu a criação do banco de dados com os elementos escolhidos para o idoso e o indivíduo com TDAH. Logo após, foi arquitetado as interfaces do software. Em seguida, iniciou-se o processo de codificação, com a criação das funções que iram manter o aplicativo, como, gerenciar usuário (cadastrar, perfil, editar e excluir); gerenciar menu para o idoso (função de aeróbicos e relaxamento, função jogos, função diário e função memória e família); e gerenciar menu para o indivíduo com TDAH (função cursos). Depois ocorreu a implantação do sistema bem como a sua utilização pelos usuários e consequentemente a análise dos resultados. Os instrumentos para a avaliação do aplicativo, foram fundamentados por meio da geração de formulários adaptados ao tema.

Desse modo, segue a listagem das ferramentas que foram utilizadas para o desenvolvimento do aplicativo e suas respectivas descrições: Ionic: framework de desenvolvimento do aplicativo; Adobe Photoshop: software para criação e edição das mídias; e Moqups online: software para a construção dos protótipos.

\section{Resultados}

No primeiro momento foi estabelecido o framework open source Ionic para a construção do aplicativo, que é um ambiente de desenvolvimento de aplicativos móveis multiplataforma, ou seja, a aplicação será executada em vários dispositivos como smartphones, iPhones, iPads, tablets, dentre outros. Este framework utiliza na estruturação do front-end, tecnologias como HTML, CSS e JavaScript e para a base de dados é empregado o Apache Cordova.

Após a instalação e configuração dos softwares, foi realizado a criação do aplicativo. A Figura 2 retrata as telas de login e do formulário para o preenchimento das informações do usuário. 
VII Congresso Brasileiro de Informática na Educação (CBIE 2018)

Anais do XXIX Simpósio Brasileiro de Informática na Educação (SBIE 2018)
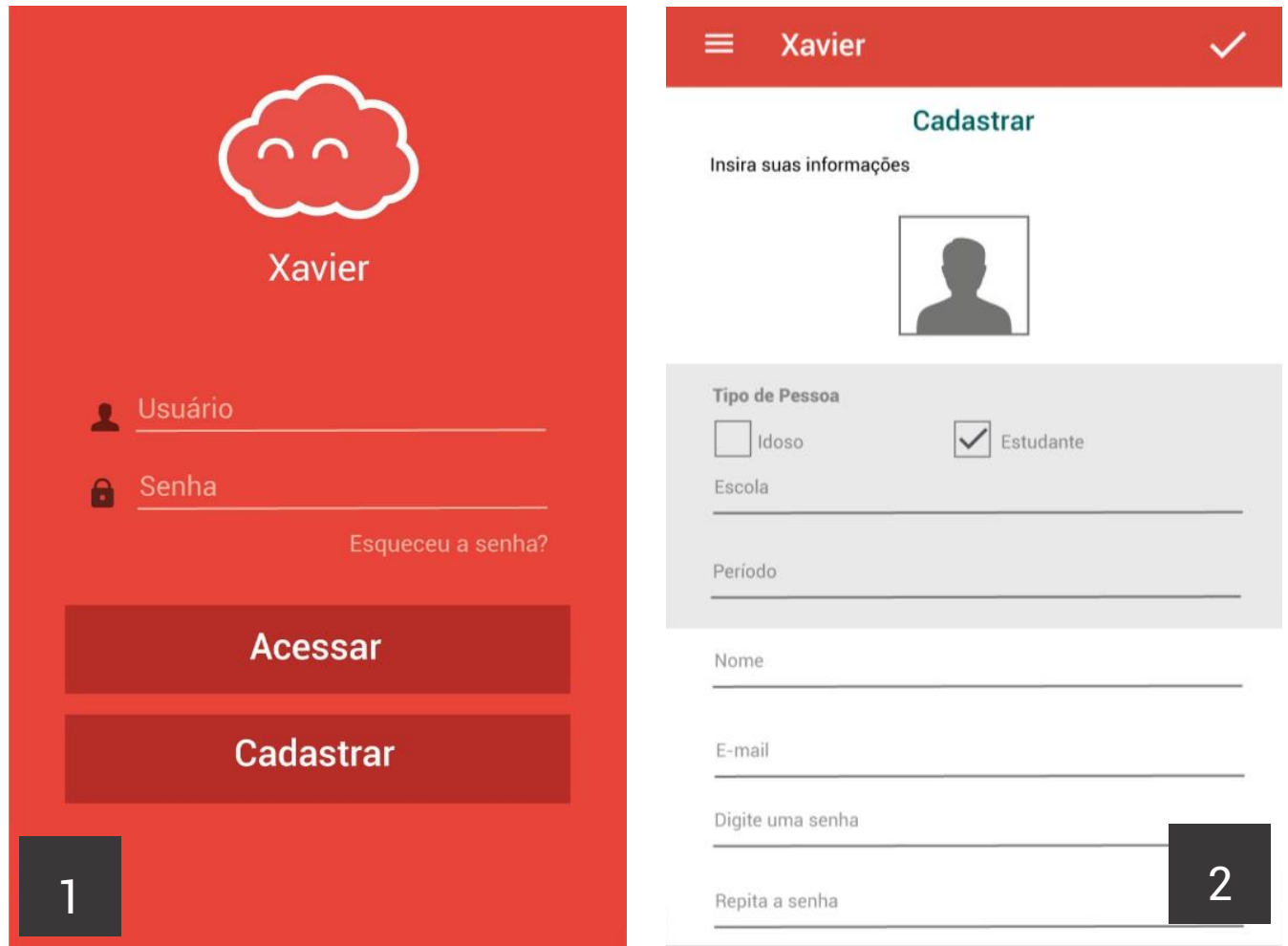

Cadastrar

Insira suas informaçōes

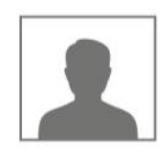

Tipo de Pessoa

$\square$ Idoso

Escola

Periodo

Nome

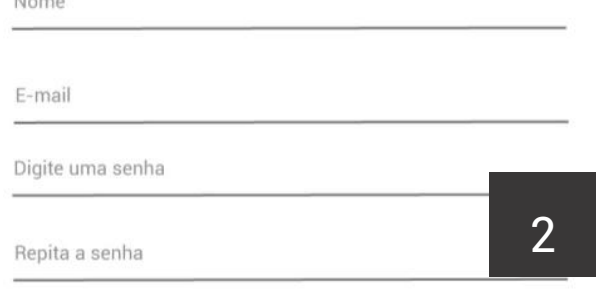

Figure 2. Login e área de cadastro do usuário. PrintScreen da tela do aplicativo

A tela de formulário de cadastro dos dados do usuário, como pode se ibservar na Figura 2 é importante porque através destas informações é possível realizar um mapeamento e estruturação dos MCE. Já a Figura 3 representa as telas de atividades para o cuidador idoso e para o indivíduo com TDAH.

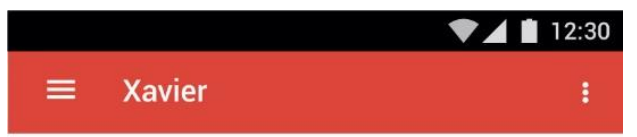

Atividades

Toque na atividade que deseja fazer

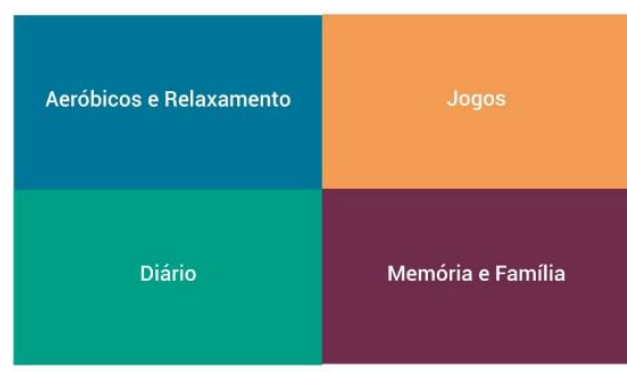

1

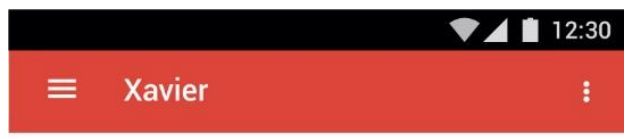

Disciplinas

Toque na disciplina que deseja fazer

\section{A}

Algoritmos e técnicas de programação I

D

Desenvolvimento Web

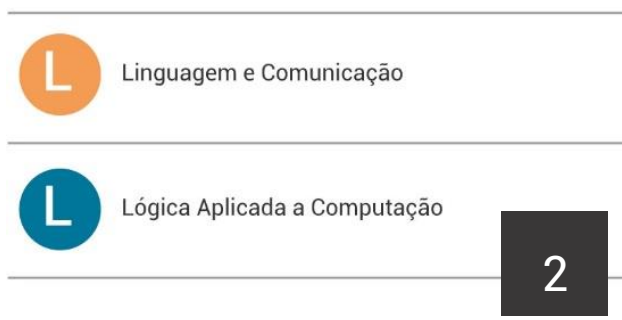

Figure 3. Ações para o cuidador e para o aluno com TDAH. PrintScreen da tela do aplicativo 
VII Congresso Brasileiro de Informática na Educação (CBIE 2018)

Anais do XXIX Simpósio Brasileiro de Informática na Educação (SBIE 2018)

A tela do menu conforme mostrado na Figura 3, é a área que o usuário irá acessar as funcionalidades do software. O item 1 da Figura 3, caracteriza a tela de menu para o idoso com Alzheimer, que contém as atividades que o idoso irá realizar. Estas atividades são: Aeróbicas e relaxamento. esta área contém sugestões destas atividades, bem como um histórico das atividades físicas que o idoso realizou; Jogos: nesta função contém sugestões de jogos; Diário: nesta opção contém dados para gerenciamento das atividades do idoso; e Memória e família: neste menu é possível obter informações sobre o que o idoso terá que recordar. No item 2 da Figura 3 indica a tela de menu do indivíduo com TDAH, que abrange as atividades das disciplinas que serão estudadas e aprendidas.

Na Figura 4 pode se visualizar a tela detalhada da atividade Memória e família, bem como de uma disciplina. Essa função é importate, pois para uma pessoal com Alzheimer os familiares podem compartilhar momentos do dia a dia com o mesmo e para o estudate o professor/tutor ou responsável pode acompanhar o seu desempenho no decorrer da disciplina.

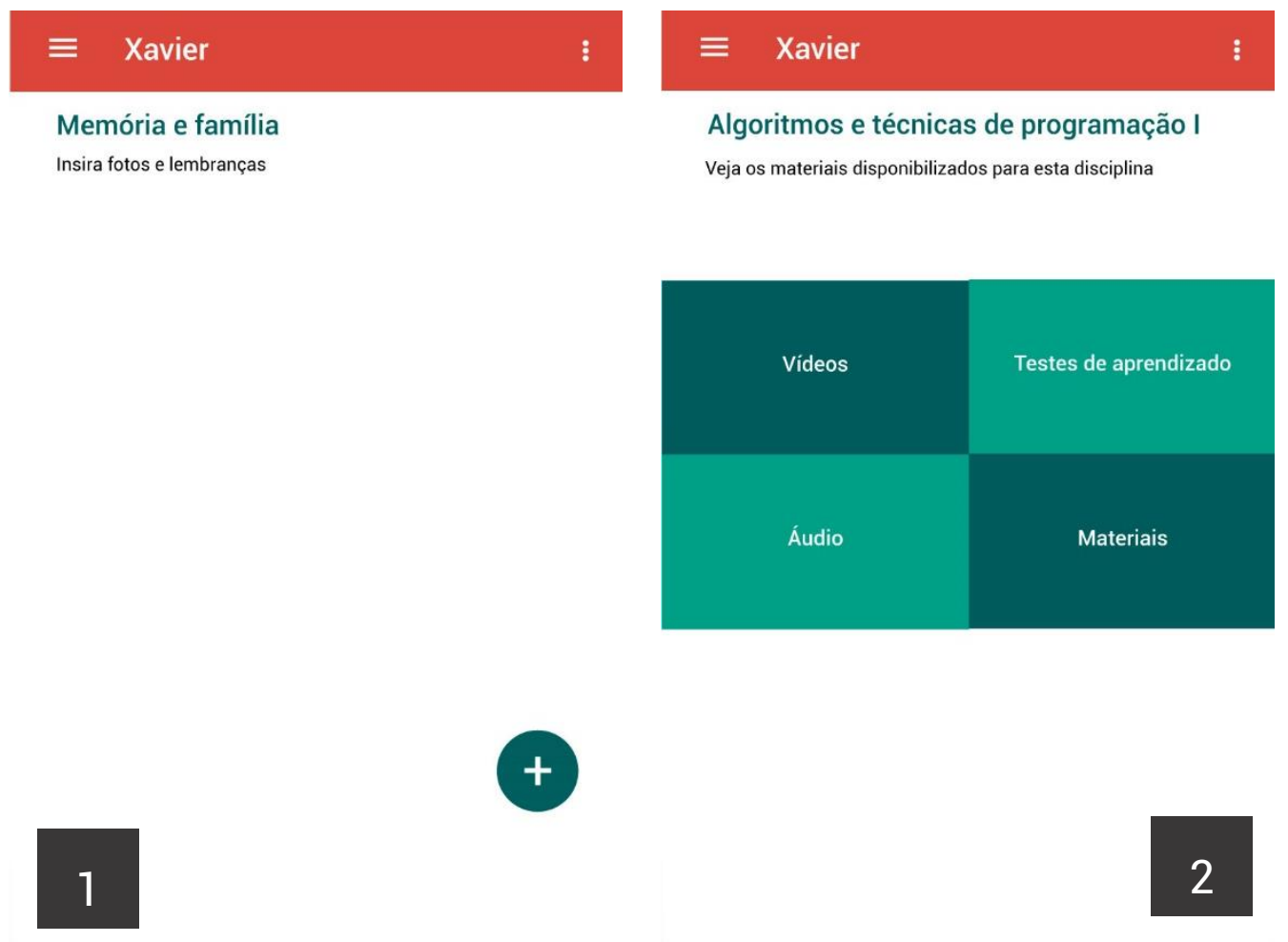

Figure 4. Memória e Família. PrintScreen da tela do aplicativo

\subsection{Utilização e avaliação}

A fase de avaliação do uso do aplicativo foi constituída através de uma análise com a população de 4 idosos com características da doença de Alzheimer e 10 alunos com TDAH de uma Instituição de Ensino Superior trabalhando as disciplinas de Algoritmos e técnicas de programação I; Desenvolvimento Web; Linguagem e Comunicação e Lógica Aplicada a Computação. O questionário incluiu itens referentes à apresentação visual, a utilização das funcionalidades disponibilizadas no aplicativo e o emprego do MMEEBB. 
VII Congresso Brasileiro de Informática na Educação (CBIE 2018)

Anais do XXIX Simpósio Brasileiro de Informática na Educação (SBIE 2018)

Entre os resultados obtidos até o momento, verificou-se que apenas 3 idosos do estudo que realizaram o MMEEBB com o uso do aplicativo tiveram mais retenção do conhecimento em sua memória. Já os indivíduos com TDAH, apenas 7 deles que conseguiram reter o conhecimento na memória. Visto que, alguns deles relataram que não seguiram corretamente o MMEEBB.

Analisou-se também que ambos os grupos acharam adequados a apresentação visual do aplicativo e a disposição das informações no mesmo. Para os alunos com TDAH foi aplicado uma prova para avaliar se realmente eles aprenderam os conteúdos. O resultado apresentou média de acertos em torno de 70 a $90 \%$ das disciplinas, comprovando o aprendizado dos estudantes.

Foram relatados pelo grupo de estudo com TDAH que a experiência foi positiva e fundamental para o aprendizado e retenção do conhecimento. Disseram ainda que, gostariam de ter mais disciplinas disponíveis no aplicativo. Isso mostra que estes estudantes estão abertos para operar tecnologias de comunicação e informação como ambiente de obtenção ao conhecimento e ao aprendizado.

\section{Considerações Finais}

A utilização dos MCE, MMEEBB e da tecnologia computacional no processo de resgate e perenização do conhecimento a idosos e pessoas com TDAH, além de disseminar os benefícios destes métodos, possibilitará a elaboração de novas ferramentas que poderão ser distribuídas e instituições de ensino e para a sociedade.

O aplicativo desenvolvido vem confirmando o efeito positivo do uso das tecnologias computacionais adaptada ao aprendizado dos indivíduos, e também do uso dos MCE e do MMEEBB nestas tecnologias, sendo indispensável a inclusão digital das pessoas. Deseja-se ainda, que o emprego de tecnologias computacionais possibilite a efetivação de metodologias mais inovadoras, favorecendo a aprendizagem mais presente dos estudantes nas atividades e também dos idosos com Alzheimer.

Portanto, a experiência de implementar estes métodos através da tecnologia computacional, foi muito significativo para transmitir o conceito e a segurança de que o uso da tecnologia e dos métodos não tem mais fronteira, basta saber utilizá-la.

\section{Referências}

Abraz (2018). O que é Alzheimer, http://www.abraz.org.br/index.php?page=alzheimer, Fevereiro.

Amorim, Cacilda (2018). TDAH - O que você precisa saber: causas, diagnóstico e tratamento, https://dda-deficitdeatencao.com.br/oquee/, Fevereiro.

Bacelar, Oscar (2009). Lembro, logo existo - principais dúvidas sobre memória e esquecimento. Rio de Janeiro: Auracom Livros.

Barkley, R. A., Anastopoulos, A. D., Robin, A. L., Lovett, B. J., Smith, B. H., Cunningham, C. E., Shapiro, C. J., Connor, D. F., DuPaul, G. J., Prince, J. B., Dooling-Litfin, J. K., Biederman, J., Murphy, K. R., Rhoads, L. H., Cunningh, L. J., Pfiffner, L. J., Gordon, M., Farley, S. E., Wilens, T. E., Spencer, T. J., Hathway, W. 
VII Congresso Brasileiro de Informática na Educação (CBIE 2018)

Anais do XXIX Simpósio Brasileiro de Informática na Educação (SBIE 2018)

(2008). Transtorno de déficit de atenção/hiperatividade: manual para diagnostico e tratamento ( $3^{\circ}$ ed.). Porto Alegre: Artmed.

Bastos, Angela Paula Zão, et al. (2012) "Utilização de um Jogo Sério e Nä̈ve Bayes para Auxiliar na Avaliação Cognitiva do Transtorno de Déficit de Atenção/Hiperatividade." Brazilian Symposium on Computers in Education (Simpósio Brasileiro de Informática na Educação-SBIE). Vol. 23. No. 1.

Collucci, Cláudia (2015). Como ampliar o alcance das inovações científicas? http://temas.folha.uol.com.br/tecnologia-em-saude/debate/como-ampliar-o-alcancedas-inovacoes-cientificas.shtml, Fevereiro.

Filho, Ronaldo Pontes Barreira and Barreira, Idalbenia V. Barbosa P. (2017) Doença de Alzheimer: Diagnóstico e Perspectivas, Gramma, p. 168.

Ferreira, Daniela Carvalho Monteiro; Lima, L. V.; Camargo Lunior, H. ; Schiovato, N. S. C. . Mapas de Conhecimento Estruturado: proposta de uma nova abordagem metodológica de ensino e aprendizagem. Educere et Educare (versão eletrônica), v. 9, p. 505-514, 2014.

Idoeta, Paula Adamo (2014). Dez tendências da tecnologia na educação, https://www.bbc.com/portuguese/noticias/2014/12/141202_tecnologia_educacao_pai , Fevereiro.

Instituto Alzheimer Brasil (2018). Doença de Alzheimer, http://www.institutoalzheimerbrasil.org.br/, Fevereiro.

Nitrini, R. (1993) Diagnóstico de demência: avaliação clínica, neuropsicológica e através da tomografia computadorizada por emissão de fóton único. Tese (livredocência). Faculdade de Medicina da Universidade de São Paulo.

Silva, Mislene Dalila da (2017). Proposta de um guia metodológico e software para quantificação qualitativa na avaliação efetiva do aprendizado em avas e ensino presencial. 76 p. Dissertação (Mestrado em Tecnologias, Comunicação e Educação) Universidade Federal de Uberlândia, Uberlândia, 2017. 